\title{
Tetracyanoethylene as a building block in the facile synthesis of heteroyl-tetrasubstituted thiazoles
}

\section{Hassan, Alaa A.}

2020-09-10

Hassan , A A , Aly , A A , Mohamed , N K , El-Haleem , L E A , Bräse , S \& Nieger , M 2020 ,

' Tetracyanoethylene as a building block in the facile synthesis of heteroyl-tetrasubstituted

thiazoles ', Monatshefte für Chemie , vol. 151 , no. 9 , pp. 1425-1431 . https://doi.org/10.1007/s00706-020-02669-4

http://hdl.handle.net/10138/334229

https://doi.org/10.1007/s00706-020-02669-4

unspecified

acceptedVersion

Downloaded from Helda, University of Helsinki institutional repository.

This is an electronic reprint of the original article.

This reprint may differ from the original in pagination and typographic detail.

Please cite the original version. 

synthesis of heteroyl-tetrasubstituted thiazoles

3 Alaa A. Hassan ${ }^{1 *}$ • Ashraf A. Aly ${ }^{1} \bullet$ Nasr K. Mohamed ${ }^{1} \bullet$ Lamiaa. E. $^{\circ}$

6 Received: ....../Accepted ...

7 Abstract The transformation of $N$-substituted 2-

8 heteroylthiosemicarbazides to $N$-(2-substituted imino)-4-amino-5-

9 cyanothiazol-3(2H)-3-yl)-heteroyl-2-carboxamides was achieved via the

10 reaction with tetracyanoethylene (TCNE). In this transformation; TCNE is

11 used as a building block. The structures of the products have been

12 confirmed unambiguously by single-crystal X-ray structure analysis. A

13 rationale for the formation of the products is presented.

14 Keywords 2,3,4,5-Tetra-substituted thiazoles - $N$-substituted 215 heteroylthiosemicarbazides $\bullet$ Heterocycles $\bullet$ Donor-acceptor effects

16 NMR spectroscopy $\bullet X$-ray structure determination.

$\bowtie$ Alaa A. Hassan

19 alaahassan2001@mu.edu.eg

$20{ }^{1}$ Chemistry Department, Faculty of Science, Minia University, El-Minia 21 61519, Egypt.

$22{ }^{2}$ Institute of Organic Chemistry, Karlsruhe Institute of Technology, Fritz23 Haber-Weg 6, Karlsruhe 76131, Germany. 
$1{ }^{3}$ Institute of Biological and Chemical Systems - Functional Molecular 2 Systems (IBCS-FMS), Karlsruhe Institute of Technology, Hermann3 von-Helmholtz-Platz 1, D-76344 Eggenstein-Leopoldshafen.

$4{ }^{4}$ Department of Chemistry, University of Helsinki, P.O. Box 55, A. I. $5 \quad$ Virtasen aukio I, Helsinki 00014, Finland. Introduction

7 Due to the accessibility and ability of carbothioamides and their 8 analogs to act as bifunctional nucleophiles [1-6], they play a significant 9 role among other nitrogen and sulfur-containing compounds.

10 Carbothioamides contain several nucleophilic centers and used as building

11 blocks for the synthesis of different heterocyclic rings [7-9].

12 Tetracyanoethylene (TCNE) is utilized as a part of a wide range of 13 reactions either as a building block or as a reaction mediator [10]. The 14 addition of nucleophiles to the double bond of TCNE followed by loss of 15 cyanated fragments was considered as the most common reaction [11-13].

16 Direct nucleophilic addition to the nitrile can also occur but is less common $17[14,15]$. Thiosemicarbazide is one of the carbothioamide derivatives. Due to

19 the presence of several nucleophilic centers in thiosemicarbazides, they are 20 also ideal candidates and valuable building blocks for the synthesis of 21 different heterocyclic rings. 
Different families of heterocyclic compounds such as thiadiazoles,

2 thiadiazines, thiadiazepines, and pyrazoles were prepared upon the reaction

3 of TCNE with 2,4-disubstituted thiosemicarbazides [16].

52 in dry ethyl acetate afforded mesoionic-1,2,4-triazolium-3-thiolate

6 derivatives 3a-c in 67-76\% yields (Scheme 1) [6], whereas upon the

7 reaction of 2-substituted hydrazinecarbothioamides with TCNE, 2-\{amino-<smiles>[R]NC(=S)NNc1ccccc1</smiles>

$$
\text { 1,3: } a, R=\mathrm{C}_{2} \mathrm{H}_{5}, \mathrm{~b}, \mathrm{R}=\mathrm{CH}_{2}-\mathrm{CH}=\mathrm{CH}_{2} ; \mathrm{c}, \mathrm{R}=\mathrm{C}_{6} \mathrm{H}_{5}
$$

11 New substituted (Z)-4-amino-3-(substituted amino)-2-(substituted

12 imino)-2,3-dihydrothiazole-5-carbonitriles and (Z)-(4-amino-5-cyano-3-

13 thiazol-2-ylidene)carbonohydrazonoyl dicyanides were synthesized via the

14 reaction of TCNE with $N$-substituted alkenylidene

15 hydrazinecarbothioamides [18]. 
The interaction between TCNE and (1-aryl ethylidene)hydrazine-

2 carbothioamides afforded 1,3-thiazine-5,6,6-tricarbonitriles and 2-thioxo-

3 2,3-dihydropyrimidine-4,5-dicarbonitriles [19]. Also, it has been reported

4 that the addition of one mole of bis-thiosemicarbazones to doubled molar

5 amounts of TCNE in dioxane gave bis-1,3-thiazines in high yields [13].

Tetracyanoethylene reacted with 1-acylthiosemicarbazides in

7 ethyl acetate with the formation of $N^{\prime}$-(4-amino-5,6-dicyano-1,3-thiazine-2-

8 ylidene)substituted hydrazide, $N$-(5-amino-3,4-dicyanopyrrol-2-ylidene)-2-

9 substituted imidazo[2,1-b][1,3,4] oxadiazole-5,6-dicarbonitriles [ 20 ].

10 On the other hand, the heterocyclization of 4-substituted-1-

11 benzoylthiosemicarbazides using TCNE afforded two kinds of ring-

12 forming reactions, i) By using DMF as a solvent of reaction, cyclization by

13 intramolecular nucleophilic attack on either the carbonyl or thiocarbonyl

14 carbon atom may be activated by TCNE to synthesize 2,5-disubstituted

15 thiadiazoles or 2,5-disubstituted oxadiazoles [11]. ii) In the less polar

16 chlorobenzene as a solvent, several possible types of tetracyanoethylation

17 adducts may be formed followed by formation pyrazole, thiadiazole, and

18 oxadiazine derivatives and TCNE reacted as a building block [11]. This

19 unique reactivity has no precedence and warrants further study. In the

20 present investigation, we undertook the preparation of less nucleophilicity

21 and electron poorer analogs of 1a-c, namely, $\mathrm{N}$-substituted 2- 
1 heteroylhydrazinecarbothioamides 4a-f and investigated their behavior

2 towards TCNE 2.

3 Results and Discussion

4 Upon addition doubled molar amounts of TCNE, 2 to solutions of

5 4a-f in dry THF with the admission of air at room temperature, the green

6 color of the transient complex was observed which gradually gives away to

7 brown and finally to a characteristic yellow color. This behavior may be

8 explained due to the initial formation of the unstable CT-complexes,

9 followed by a chemical reaction. The mixture was stirred and then left to

10 stand for $72 \mathrm{~h}$ at room temperature. After complete, the reaction

11 concentrated the residue was subjected to chromatographic separation.

12 From the one significant zone and by crystallization, yellow to orange

13 crystals of $\mathrm{N}$-(2-substituted imino)-4-amino-5-cyanothiazol-3(2H)-3-yl)-

14 heteroyl-2-carboxamides 5a-f were obtained in 79-68\% (Scheme 2).

15 The structure assignments of 5a-f were delineated from their

16 spectroscopic properties, elemental analyses, and single-crystal X-ray

17 crystallography. The molecular structure of $\mathbf{5 a}$, as an example, was

18 supported by the following findings: the molecular formula of $\mathbf{5 a}$

$19\left(\mathrm{C}_{17} \mathrm{H}_{14} \mathrm{~N}_{6} \mathrm{OS}\right)$ represented a product from one molecule of $4 \mathbf{a}$ and one

20 molecule of TCNE (2) with loss one molecule of malononitrile with 
1 abstraction two hydrogens from the start $\mathbf{4 a}$ and giving rise to the ion $\mathrm{m} / \mathrm{z}$

2350.

$3 \quad$ Scheme 2<smiles>[R]N=c1sc(C#N)c(N)n1NC(=O)NCCC</smiles>

The IR spectrum of 5a shows characteristic absorption bands for the

$6 \mathrm{NH}_{2}$ and amide-NH groups at 3187-3305 and 2189 for cyano group, 1701

7 and $1633 \mathrm{~cm}^{-1}$ for amide $\mathrm{C}=\mathrm{O}$ and $\mathrm{C}=\mathrm{N}$ groups, The ${ }^{1} \mathrm{H}$ NMR spectra of $\mathbf{5 a}$

8 clearly shows the presence of $\mathrm{NH}_{2}$ group by broad signal at 7.45 for two

9 protons. Compound 5a clearly shows the presence of broad amide-NH at

$1011.30 \mathrm{ppm}$ (one proton) and two protons for benzyl- $\mathrm{CH}_{2}$ as a singlet signal

11 at $4.21 \mathrm{ppm}$, in addition to nine aryl protons as multiplets in the range 7.12-

$128.76 \mathrm{ppm}$. In the ${ }^{13} \mathrm{CNMR}$ spectrum of $\mathbf{5 a}$ the thiazole $\mathrm{C}-4, \mathrm{C}-5$ resonated

13 at 161.65 , and 55.83 were by the observed trends for chemical shifts values

14 of carbon atoms in push-pull alkenes [21, 22]. The presence of a $\mathrm{Ph}-\mathrm{CH}_{2}$

15 group is also evident from the ${ }^{13} \mathrm{C}$-DEPT-NMR spectrum exhibiting a

16 negative signal at $57.7 \mathrm{ppm}$. Whereas, thiazole-C5 in compound 5a was

17 evident from the ${ }^{13} \mathrm{C}$ NMR-DEPT spectra exhibiting positive signals at $\delta_{\mathrm{C}}=$ 
$155.58 \mathrm{ppm}$. The presence of an isothiourea carbon at 153.6 and amide $\mathrm{C}=\mathrm{O}$

2 at $163.88 \mathrm{ppm}$ was supported by ${ }^{13} \mathrm{C}-\mathrm{NMR}$.

3 The structure of 5a-f was confirmed by single-crystal X-ray 4 crystallography. The structure of $N$-(2-allyl imino)-4-amino-55 cyanothiazol-3(2H)-3-yl)-furan-2-carboxamide (5d) was determined by X6 ray analysis and crystallized from ethanol in triclinic space group P-1 (Fig.

7 1, Tables S2-S8 in supplementary data).

8 The $\mathrm{C}(2)-\mathrm{S}(1)$ and $\mathrm{C}(5)-\mathrm{S}(1)$ bond lengths of $1.7684(12) \AA$ and $9 \quad 1.7717(13) \AA$ respectively due to C-S $\sigma$-bond. The $\mathrm{C}(2)-\mathrm{N}(21)$ bond length 10 of 1.2605 (17) $\AA$ and C(4)-C(5) bond length of 1.3730 (17) $\AA$ are closed to $11 \mathrm{C} / \mathrm{C}$ double bonds, the thiazole ring is planar (mean deviation from the 12 thiazole plane $0.017 \AA$ A). The same structures were assumed for other 13 derivatives based on their NMR spectroscopic similarities.

14

15

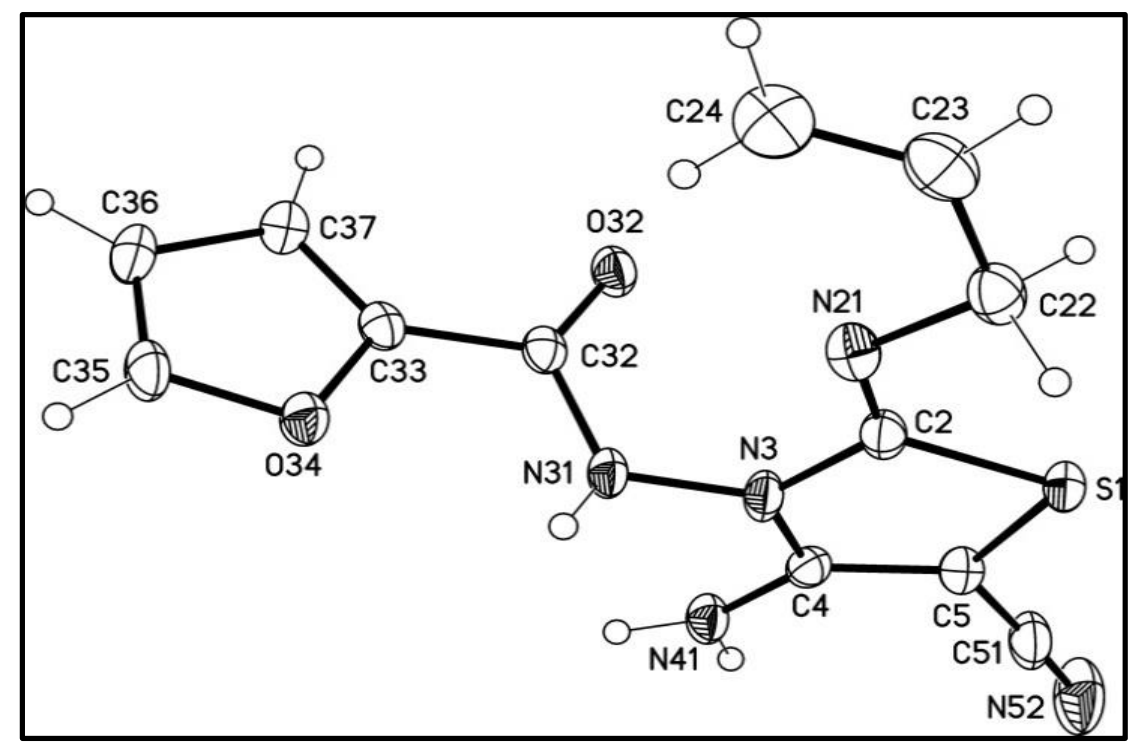

Fig. 1. Molecular structure of $\mathbf{5 d}$ (displacement parameters are drawn at $50 \%$ probability level. 
1 With the optimized reaction conditions in hand, an increased amount

2 of the start 4a-f was not necessary for the formation of high yields of the

3 products 5a-f, whereas upon addition two equivalents of TCNE, tetra-

4 substituted thiazole derivatives 5a-f were separated. Different solvents such

5 as methylene chloride, benzene, acetonitrile, and 1,4-dioxane were studied

6 but THF proved to be the best. The high yields of the products were

7 obtained at room temperature and in the presence of air, where trace

8 amounts of products were formed when the reaction subjected under

9 nitrogen or argon.

Table1, Yields of synthetic products 5a-f in different solvents.

\begin{tabular}{|c|c|c|c|c|c|c|}
\hline \multirow{2}{*}{$\begin{array}{c}\text { Compound } \\
\text { number }\end{array}$} & \multirow[t]{2}{*}{ Structure } & \multicolumn{5}{|c|}{ Yield of 5a-f (\%) } \\
\hline & & THF & $\mathrm{CH}_{2} \mathrm{Cl}_{2}$ & Benzene & $\mathrm{CH}_{3} \mathrm{CN}$ & 1,4-dioxane \\
\hline $5 \mathbf{a}$ & & 79 & 36 & 33 & 40 & 56 \\
\hline \multirow[t]{2}{*}{$5 b$} & & & & & & \\
\hline & & 71 & 29 & 30 & 38 & 53 \\
\hline
\end{tabular}




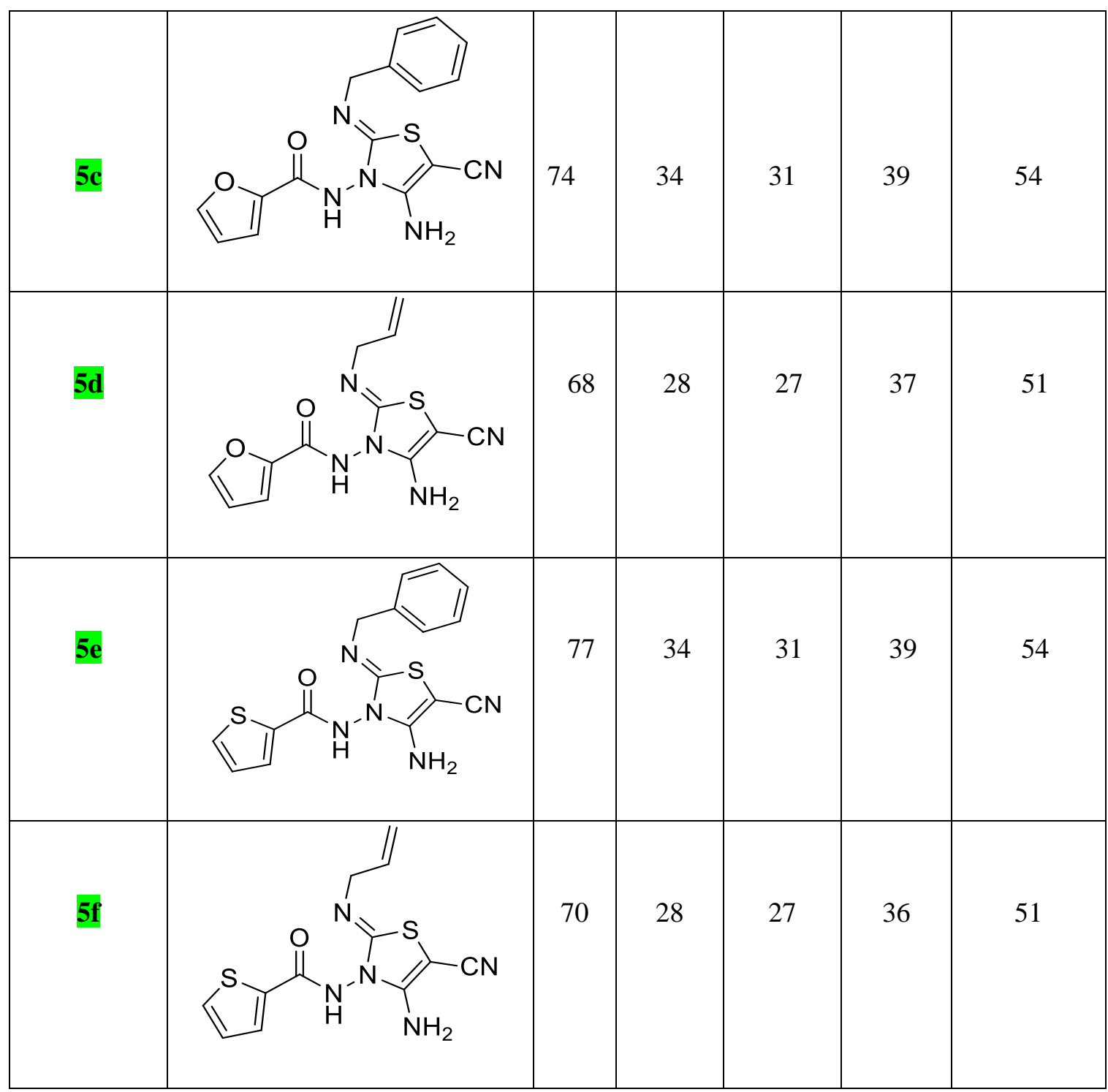

Mechanistic rationale for the formation of tetra-substituted thiazoles

3 5a-f as depicted in Scheme 3.

4 Charge transfer complexes may (but not necessarily have to) play an

5 intermediate role. Two routes may be suggested after the formation of the

6 radicals $4^{\circ}$ and $2^{\circ} \mathrm{H}$. In the first route, the combination of the two radicals

7 afforded the intermediate 6a-f. Elimination of a molecule of $\mathrm{CH}_{2}(\mathrm{CN})_{2}$

8 with the abstraction of another hydrogen from 4 gave the intermediate $7 \mathbf{a}-\mathbf{f}$ 
1 which abstracts another proton of $\mathbf{4}$ to give the imine $\mathbf{8}$. Cyclization of the

2 imine 8 via intramolecular nucleophilic attack of hydrazide $\mathrm{NH}$ on the

3 imine $\mathrm{C}=\mathrm{NH}$ afforded tetra-substituted thiazoles 5a-f.

4 In the second route, the cyclizations involve intramolecular

5 nucleophilic attacks on either a thiocarbonyl or carbonyl group (scheme 3).

6 Since the aforementioned reactions do not take place when 2 are added to

7 4a-f, this means that TCNE, 2 was used as a building block in this reaction 8 and not a mediator.

\section{Conclusion}

10 In conclusion, novel heteroyl-tetrasubstituted thiazole derivatives

11 containing aliphatic and aromatic substituents are presented via cyclization

12 of $\mathrm{N}$-substituted 2-heteroylhydrazinecarbothioamides by reaction with

13 TCNE. Heteroylhydrazinecarbothioamides are multi-dentate nucleophiles

14 allowing for various modes of heterocyclization with TCNE, providing 15 several electrophilic sites.

\section{Experimental}

17 Melting points (mp's) were determined using open glass capillaries 18 on a Gallenkamp melting point apparatus (Gallenkamp, UK) and are

19 uncorrected. The IR spectra were recorded with alpha Bruker FT-IR a

20 Bruker instrument using $\mathrm{KBr}$ pellets. $400{ }^{1} \mathrm{H}$ NMR and $100{ }^{13} \mathrm{C}$-NMR were

21 determined on a Bruker AM400 spectrometer with tetramethylsilane as 


$$
\text { 4a-f }+2 \rightleftharpoons[\text { CT-complex }] \rightleftarrows\left[\dot{\bullet}^{+}+\ddot{2}_{\mathbf{2}}^{-}\right] \longrightarrow[\dot{\mathbf{4}}+2 \dot{\mathrm{H}}] \longrightarrow
$$

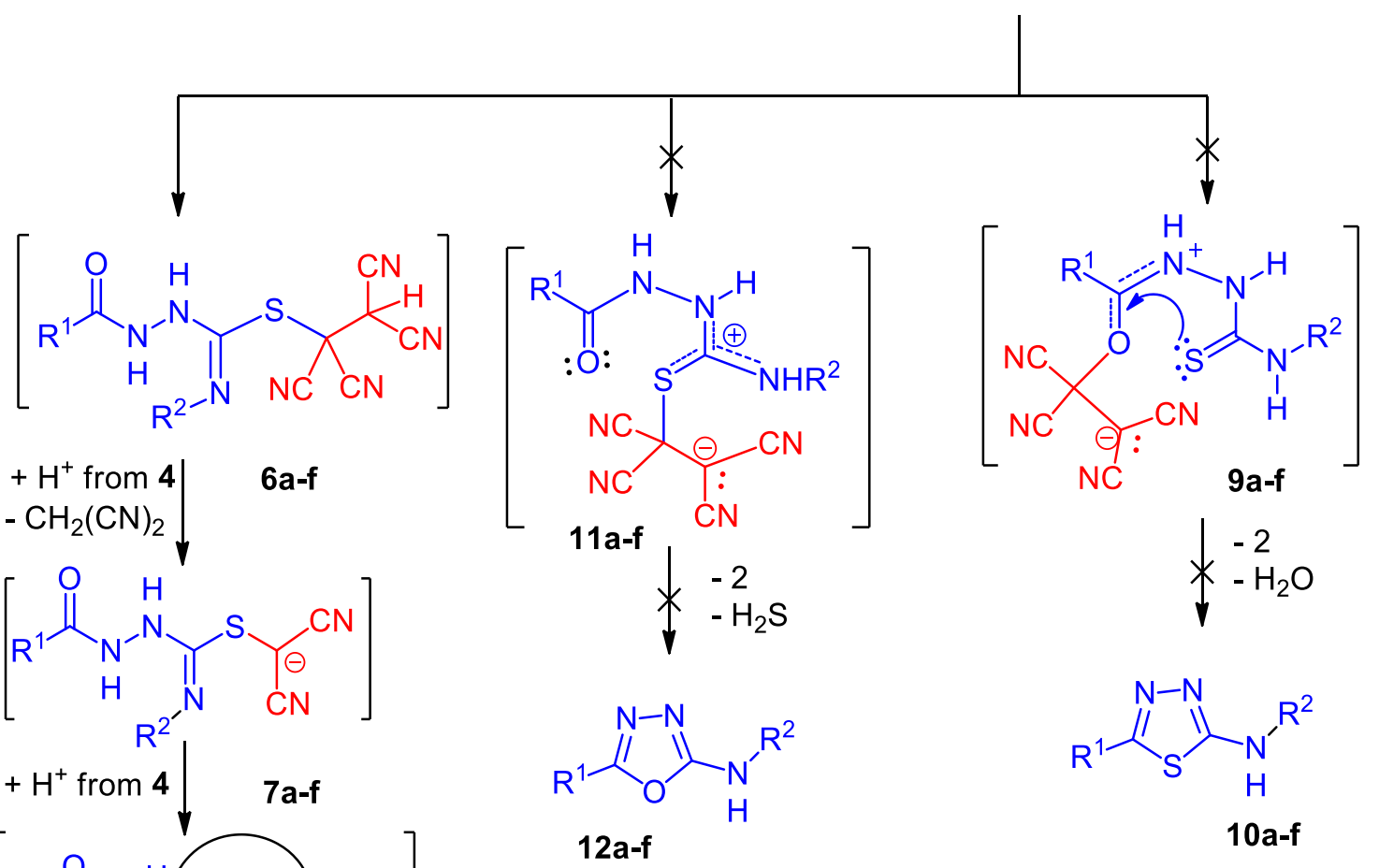<smiles>[R]N=c1sc(C#N)c(N)n1NC([R])=O</smiles>

3 internal standard; $\mathrm{s}=$ singlet, $\mathrm{m}=$ multiplet, $\mathrm{br}=$ broad. The ${ }^{13} \mathrm{C}$ NMR

4 signals were assigned based on DEPT 135/90 spectra, chemical shifts are

5 expressed as $\delta$. The mass spectra $(70 \mathrm{eV}$, electron impact mode were

6 recorded on a Finnigan MAT 312 instrument). The elemental analyses for

$7 \mathrm{C}, \mathrm{H}, \mathrm{N}$, and $\mathrm{S}$ were carried out on a Carlo Erba model $1106 \mathrm{C}, \mathrm{H}, \mathrm{N}$, and S

8 analyzer. Preparative layer chromatography (Plc) used air-dried $1.0 \mathrm{~mm}$ 


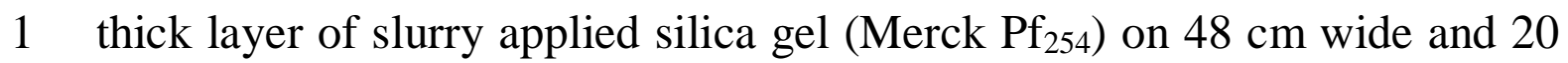

$2 \mathrm{~cm}$ high glass plates using the solvent listed.

\section{Starting materials}

$4 \quad \mathrm{~N}$-substituted 2-heteroylhydrazinecarbothioamides 4a-f were prepared 5 according to the literature. $\mathbf{4 a}, \mathbf{4 b}, \mathbf{4 d}, \mathbf{4 f}[23], \mathbf{4 b}, \mathbf{4 c}$ [24].

6 Tetracyanoethylene (ethenetetracarbonitrile, 2, Merck) was recrystallized 7 from chlorobenzene and sublimed.

8 Reactions of $N$-substituted 2-heteroylthiosemicarbazides 4a-f with

9 TCNE 2

10 General procedure: A solution of $N$-substituted 2-heteroylthiosemi-

11 carbazides 4 a-f $(1.0 \mathrm{mmol})$ in dry THF $(25 \mathrm{ml})$ was added dropwise with

12 stirring at room temperature to $2.0 \mathrm{mmol}$ of TCNE 2 in $(20 \mathrm{ml})$ THF. The

13 reaction mixture was stirred followed by TLC for $72 \mathrm{hrs}$ at room

14 temperature which caused a spontaneous change of color from green to

15 brown and finally to yellow with consuming all the start materials and 16 complete the reaction. After concentration to dryness, the residue was

17 sublimed at $80{ }^{\circ} \mathrm{C}$ under vacuum to remove the excess of TCNE and then 18 subjected to preparative thin-layer chromatography using toluene /ethyl 19 acetate (1:1) in case of the reaction with $(\mathbf{4 c}, \mathbf{4 d}, \mathbf{4 e}$, and $\mathbf{4 f})$ and 20 toluene/ethyl acetate (1:2) in case of (4a and $\mathbf{4 b}$ ) as eluent to give

21 numerous colored zones. Only one high intense zone was removed and 
1 extracted with acetone then recrystallized to give pure crystals of 5a-f. The

2 other colored zones were very small.

3 (Z)- $N$-(4-Amino-2-(benzylimino)-5-cyanothiazol-3(2H)-yl)picolinamide $4 \quad\left(5 \mathrm{a}, \mathrm{C}_{17} \mathrm{H}_{14} \mathrm{~N}_{6} \mathrm{OS}\right)$

5 Yellow crystals (acetonitrile), $\left(\mathrm{R}_{\mathrm{f}}=0.27\right)$, m.p.: $124-125^{\circ} \mathrm{C}$; yield: $276 \mathrm{mg}$ $6(79 \%) ; \quad$ IR $(\mathrm{KBr}) \bar{\nu}=3305\left(\mathrm{NH}_{2}\right), 3187(\mathrm{NH}), 3082(\mathrm{Ar}-\mathrm{CH}), 2189$ $7(\mathrm{C} \equiv \mathrm{N}), 1701(\mathrm{C}=\mathrm{O}), 1623(\mathrm{C}=\mathrm{N}), 1585(\mathrm{Ar}-\mathrm{C}=\mathrm{C}) \mathrm{cm}^{-1} ;{ }^{1} \mathrm{H}$ NMR $(400$ $\left.8 \mathrm{MHz}, \mathrm{DMSO}-d_{6}\right): \delta=11.30(\mathrm{~s}, 1 \mathrm{H}$, amide- NH), 8.76-8.66 (m, 1H, Pyr-

9 H), 8.10-7.99 (m, 2H, Pyr-H), 7.70-7.66 (m, 1H, Pyr-H), 7.45 (s, 2H, $\mathrm{NH}_{2}$

10 attached to thiazole), 7.35-7.12 (m, 5H, Ph-H), $4.21\left(\mathrm{~s}, 2 \mathrm{H}, \mathrm{CH}_{2} \mathrm{Ph}\right) \mathrm{ppm}$;

$11{ }^{13} \mathrm{C}$ NMR (100 MHz, DMSO- $\left.d_{6}\right): \delta=163.88$ (amide-CO), 161.65

(thiazole-C4), 153.6 (thiazole-C2), 150.6 (Pyr-C), 148.8 (Pyr-CH), 139.5

13 (Pyr-CH), 138.0 (Ph-C), 128.3 (Ph-2CH), 127.7 (Ph-CH), 127.3 (Ph-2CH),

14 126.7, $123.0(\mathrm{Pyr}-\mathrm{CH}), 116.8(\mathrm{C} \equiv \mathrm{N}), 57.7\left(\mathrm{CH}_{2} \mathrm{Ph}\right), 55.83$ (thiazole-C5)

15 ppm; MS (FAB) $m / z(\%)=350\left(\mathrm{M}^{+}, 100\right)$; Calcd: C, 58.27; H, 4.03; N, $16 \quad 23.98 ; \mathrm{S}, 9.15$.

17 (Z)- $N$-(2-(Allylimino)-4-amino-5-cyanothiazol-3(2H)-yl)picolinamide $18\left(\mathbf{5 b}, \mathbf{C}_{13} \mathrm{H}_{12} \mathbf{N}_{6} \mathrm{OS}\right)$

19 Yellow crystals (acetonitrile), $\left(\mathrm{R}_{\mathrm{f}}=0.29\right)$, m.p.: $170-17{ }^{\circ} \mathrm{C}$; yield: 213 $20 \mathrm{mg}(71 \%) ; \mathrm{IR}(\mathrm{KBr}) \bar{\nu}=3284\left(\mathrm{NH}_{2}\right), 3185(\mathrm{NH}), 3066(\mathrm{Ar}-\mathrm{CH}), 2191$ $21(\mathrm{C} \equiv \mathrm{N}), 1700(\mathrm{C}=\mathrm{O}), 1630(\mathrm{C}=\mathrm{N}), 1589(\mathrm{Ar}-\mathrm{C}=\mathrm{C}) \mathrm{cm}^{-1} ;{ }^{1} \mathrm{H}$ NMR $(400$ 
$\left.1 \mathrm{MHz}, \mathrm{DMSO}-d_{6}\right): \delta=11.25(\mathrm{~s}, 1 \mathrm{H}$, amide- NH), $8.83-8.62(\mathrm{~m}, 1 \mathrm{H}, \mathrm{Pyr}-$

2 H), 8.21-7.92 (m, 2H, Pyr-H), 7.72-7.66 (m, 1H, Pyr-H), 7.43 (s, 2H, $\mathrm{NH}_{2}$

3 attached to thiazole $), 5.85-5.76(\mathrm{~m}, 1 \mathrm{H}$, allyl-CH=), 5.40-4.87 (m, 2H,

4 allyl- $\left.\mathrm{CH}_{2}=\right)$, 3.66-3.62 (m, 2H, allyl- $\left.\mathrm{CH}_{2} \mathrm{~N}\right) \mathrm{ppm} ;{ }^{13} \mathrm{C} \mathrm{NMR}(100 \mathrm{MHz}$,

5 DMSO- $d_{6}$ ): $\delta=164.1$ (amide-CO), 153.6 (thiazole-C4) 150.3 (Pyr-C),

6148.9 (Pyr-CH), 148.8 (thiazole-C2), 138.1 (Pyr-CH), 135.3 (allyl-CH=),

$7127.8,123.1\left(\right.$ Pyr-CH), $116.9\left(\right.$ allyl- $\left.\mathrm{CH}_{2}=\right), 115.4(\mathrm{C} \equiv \mathrm{N}), 55.0$ (thiazole-

8 C5), 43.6(allyl- $\left.\mathrm{CH}_{2}\right)$ ppm; MS (FAB) $m / z(\%)=300\left(\mathrm{M}^{+}, 100\right)$; Calcd: $\mathrm{C}$,

$9 \quad 51.99 ; \mathrm{H}, 4.03 ; \mathrm{N}, 27.98 ; \mathrm{S}, 10.68$.

10 (Z)- $N$-(4-Amino-2-(benzylimino)-5-cyanothiazol-3(2H)-yl)furan-2-

11 carboxamide $\left(5 \mathrm{c}, \mathrm{C}_{16} \mathrm{H}_{13} \mathrm{~N}_{5} \mathrm{O}_{2} \mathrm{~S}\right)$

12 Orange crystals (acetonitrile), $\left(\mathrm{R}_{\mathrm{f}}=0.44\right)$, m.p.: $180-181{ }^{\circ} \mathrm{C}$; yield: 250

$13 \mathrm{mg}(74 \%) ; \mathrm{IR}(\mathrm{KBr}) \bar{\nu}=3310\left(\mathrm{NH}_{2}\right), 3191(\mathrm{NH}), 3045(\mathrm{Ar}-\mathrm{CH}), 2190$

$14(\mathrm{C} \equiv \mathrm{N}), 1695(\mathrm{C}=\mathrm{O}), 1627(\mathrm{C}=\mathrm{N}), 1582(\mathrm{Ar}-\mathrm{C}=\mathrm{C}) \mathrm{cm}^{-1} ;{ }^{1} \mathrm{H}$ NMR $(400$

$\left.15 \mathrm{MHz}, \mathrm{DMSO}-d_{6}\right): \delta=11.10(\mathrm{~s}, 1 \mathrm{H}$, amide-NH), 7.98-7.88 (m, 1H, Furan-

$16 \mathrm{H}), 7.75$ (s, 2H, $\mathrm{NH}_{2}$ attached to thiazole), 7.69-7.30 (m, 5H, Ph-H), 7.28-

$177.10\left(\mathrm{~m}, 2 \mathrm{H}\right.$, Furan-H), $4.32\left(\mathrm{~s}, 2 \mathrm{H}, \mathrm{CH}_{2} \mathrm{Ph}\right) \mathrm{ppm} ;{ }^{13} \mathrm{C}$ NMR (100 MHz,

18 DMSO- $d_{6}$ ): $\delta=157.3$ (amide-CO), 153.3 (thiazole-C4), 145.5 (Furan-C2),

19139.2 (Furan-CH), 146.3 (thiazole-C2), $139.0(\mathrm{Ph}-\mathrm{C}), 128.1,127.1(\mathrm{Ph}-$

$202 \mathrm{CH}), 126.5(\mathrm{Ph}-\mathrm{CH}), 116.4(\mathrm{C} \equiv \mathrm{N}), 116.1,113.1 \quad$ (Furan-CH), 57.6 
1 (thiazole-C5), $55.8\left(\mathrm{CH}_{2} \mathrm{Ph}\right) \mathrm{ppm}$; MS (FAB) $\mathrm{m} / z(\%)=339\left(\mathrm{M}^{+}, 100\right)$;

2 Calcd: C, 56.63; H, 3.86; N, 20.64; S, 9.45.

3 (Z)- $N$-(2-(Allylimino)-4-amino-5-cyanothiazol-3(2H)-yl)furan-2-

4 carboxamide (5d, $\left.\mathrm{C}_{12} \mathrm{H}_{11} \mathrm{~N}_{5} \mathrm{O}_{2} \mathrm{~S}\right)$

5 Yellow crystals (acetonitrile), $\left(\mathrm{R}_{\mathrm{f}}=0.42\right)$, m.p.: $130-131^{\circ} \mathrm{C}$; yield: $196 \mathrm{mg}$ $6(68 \%), \mathrm{IR}(\mathrm{KBr}) \bar{\nu}=3305\left(\mathrm{NH}_{2}\right), 3181(\mathrm{NH}), 3060(\mathrm{Ar}-\mathrm{CH}), 2195(\mathrm{C} \equiv \mathrm{N})$,

$71700(\mathrm{C}=\mathrm{O}), 1623(\mathrm{C}=\mathrm{N}), 1590(\mathrm{Ar}-\mathrm{C}=\mathrm{C}) \mathrm{cm}^{-1} ;{ }^{1} \mathrm{H}$ NMR $(400 \mathrm{MHz}$,

8 DMSO- $\left.d_{6}\right): \delta=11.20$ (s, 1H, amide-NH), 8.28-8.02 (m, 1H, Furan-H),

$97.58-7.30$ (m, 2H, Furan-H), 7.25 (s, 2H, $\mathrm{NH}_{2}$ attached to thiazole), 5.95-

$105.73\left(\mathrm{~m}, 1 \mathrm{H}\right.$, allyl-CH=), 5.47-4.80 (m, 2H, allyl- $\left.\mathrm{CH}_{2}=\right), 3.96-3.61(\mathrm{~m}$,

$112 \mathrm{H}$, allyl- $\left.\mathrm{CH}_{2}\right) \mathrm{ppm} ;{ }^{13} \mathrm{C}$ NMR $\left(100 \mathrm{MHz}, \mathrm{DMSO}-d_{6}\right): \delta=158.3$ (amide-

12 CO), 154.3 ((thiazole-C4), 145.3 (Furan-C2), 139.3 (Furan-CH), 147.3

13 (thiazole-C2), 133.3 (allyl-CH=), $117.2\left(\right.$ allyl- $\left.\mathrm{CH}_{2}=\right), 116.7(\mathrm{C} \equiv \mathrm{N}), 116.2$,

14114.1 (Furan-CH), 56.8 (thiazole-C5), $48.6\left(\right.$ allyl- $\left.\mathrm{CH}_{2}\right)$ ppm; MS (FAB)

$15 \mathrm{~m} / \mathrm{z}(\%)=289\left(\mathrm{M}^{+}, 50\right)$; Calcd: $\mathrm{C}, 49.82 ; \mathrm{H}, 3.83 ; \mathrm{N}, 24.21 ; \mathrm{S}, 11.08$.

16 (Z)- $N$-(4-Amino-2-(benzylimino)-5-cyanothiazol-3(2H)-yl)thiophene-2-

17 carboxamide (5e, $\left.\mathbf{C}_{16} \mathrm{H}_{13} \mathbf{N}_{5} \mathrm{OS}_{2}\right)$

18 Orange crystals (acetonitrile), $\left(\mathrm{R}_{\mathrm{f}}=0.51\right)$, m.p.: $140-141^{\circ} \mathrm{C}$; yield: $273 \mathrm{mg}$ 19 (77\%); IR $(\mathrm{KBr}) \bar{\nu}=3308\left(\mathrm{NH}_{2}\right), 3171(\mathrm{NH}), 3070(\mathrm{Ar}-\mathrm{CH}), 2186$ $20(\mathrm{C} \equiv \mathrm{N}), 1690(\mathrm{C}=\mathrm{O}), 1630(\mathrm{C}=\mathrm{N}), 1562(\mathrm{Ar}-\mathrm{C}=\mathrm{C}) \mathrm{cm}^{-1} ;{ }^{1} \mathrm{H}$ NMR $(400$ 
$\left.1 \mathrm{MHz}, \mathrm{DMSO}-d_{6}\right): \delta=11.25(\mathrm{~s}, 1 \mathrm{H}$, amide-NH), $8.01-7.78(\mathrm{~m}, 3 \mathrm{H}$,

2 Thiophene- $\mathrm{H}), 7.55\left(\mathrm{~s}, 2 \mathrm{H}, \mathrm{NH}_{2}\right.$ attached to thiazole), $7.42-7.02(\mathrm{~m}, 5 \mathrm{H}$,

$3 \mathrm{Ph}-\mathrm{H}), 4.29\left(\mathrm{~s}, 2 \mathrm{H}, \mathrm{CH}_{2} \mathrm{Ph}\right) \mathrm{ppm} ;{ }^{13} \mathrm{C} \mathrm{NMR}\left(100 \mathrm{MHz}, \mathrm{DMSO}-d_{6}\right): \delta=$

4161.1 (amide CO), 153.3 (thiazole-C4), 150.6 (thiazole-C2), 139.3

5 (thiophene-C2), 135.9 (Ph-C), 132.6, 130.5 (thiophene-CH), 128.1, 127.1

$6 \quad(\mathrm{Ph}-2 \mathrm{CH}), 127.0 \quad(\mathrm{Ph}-\mathrm{CH}), 126.5 \quad$ (thiophene- $\mathrm{CH}), 116.4(\mathrm{C} \equiv \mathrm{N}), 63.4$

7 (thiazole-C5), $55.8\left(\mathrm{CH}_{2} \mathrm{Ph}\right) \mathrm{ppm}$; $\mathrm{MS}(\mathrm{FAB}) \mathrm{m} / z(\%)=355\left(\mathrm{M}^{+}, 80\right)$;

8 Calcd: C, 54.07; H, 3.69; N, 19.70; S, 18.04.

9 (Z)-N-(2-(Allylimino)-4-amino-5-cyanothiazol-3(2H)-yl)thiophene-2-

10 carboxamide (5f, $\left.\mathbf{C}_{12} \mathrm{H}_{11} \mathbf{N}_{5} \mathbf{O S}_{2}\right)$

11 Yellowish orange crystals (acetonitrile), $\left(\mathrm{R}_{\mathrm{f}}=0.53\right)$, m.p.: $152-153{ }^{\circ} \mathrm{C}$;

12 yield: $213 \mathrm{mg}(70 \%)$; IR $(\mathrm{KBr}) \bar{\nu}=3295\left(\mathrm{NH}_{2}\right), 3180(\mathrm{NH}), 3058(\mathrm{Ar}-$

$13 \mathrm{CH}), 2185(\mathrm{C} \equiv \mathrm{N}), 1690(\mathrm{C}=\mathrm{O}), 1625(\mathrm{C}=\mathrm{N}), 1526(\mathrm{Ar}-\mathrm{C}=\mathrm{C}) \mathrm{cm}^{-1} ;{ }^{1} \mathrm{H}$

14 NMR (400 MHz, DMSO- $\left.d_{6}, \mathrm{ppm}\right) \delta=11.20$ (br, $1 \mathrm{H}$, amide- NH), 8.10$157.82\left(\mathrm{~m}, 3 \mathrm{H}\right.$, Thiophene-H), $7.55\left(\mathrm{~s}, 2 \mathrm{H}, \mathrm{NH}_{2}\right.$ attached to thiazole $), 5.90-$ $165.74(\mathrm{~m}, 1 \mathrm{H}$, allyl- $\mathrm{CH}=), 5.21-4.99\left(\mathrm{~m}, 2 \mathrm{H}\right.$, allyl- $\left.\mathrm{CH}_{2}=\right), 3.81-3.68(\mathrm{~m}$, $172 \mathrm{H}$, allyl- $\left.\mathrm{CH}_{2} \mathrm{~N}\right) \cdot{ }^{13} \mathrm{C}$ NMR $\left(100 \mathrm{MHz}, \mathrm{DMSO}-d_{6}, \mathrm{ppm}\right) \delta=160.1$ (amide$18 \mathrm{CO}$ ), 154.4 (thiazole-C4), 138.3 (thiazole-C2), 137.2 (thiophene-C2), $19133.7,132.2$ (thiophene- $\mathrm{CH}$ ), 131.3 (allyl-CH=), 129.3 (thiophene-CH), $20117.4\left(\right.$ allyl- $\left.\mathrm{CH}_{2}=\right), 114.6(\mathrm{C} \equiv \mathrm{N}) 58.3$ (thiazole-C5), $49.6\left(\right.$ allyl- $\left.\mathrm{CH}_{2} \mathrm{~N}\right)$. 
1 MS (FAB) $m / z(\%)=305\left(\mathrm{M}^{+}, 60\right)$; Calcd: $\mathrm{C}, 47.20 ; \mathrm{H}, 3.63 ; \mathrm{N}, 22.93 ; \mathrm{S}$, 221.00.

\section{Crystal Structure Determination of 5d}

4 The single-crystal X-ray diffraction study was carried out on a Bruker D8

5 Venture diffractometer with PhotonII detector at $123 \mathrm{~K}$ using $\mathrm{Cu}-\mathrm{K} \alpha$ 6 radiation $(\lambda=1.54178 \AA$ ). Dual space Methods (SHELXT) [25] were used

7 for structure solution and refinement was carried out using SHELXL-2013 8 / 2014 (full-matrix least-squares on $F^{2}$ ) [26]. Hydrogen atoms were 9 localized by difference Fourier synthesis map and refined using a model $10(\mathrm{H}(\mathrm{N})$ free $)$. Semi-empirical absorption correction was applied.

11 Compound 5d: $\mathrm{C}_{12} \mathrm{H}_{11} \mathrm{~N}_{5} \mathrm{O}_{2} \mathrm{~S}, \mathrm{Mr}=289.32 \mathrm{~g} \mathrm{~mol}^{-1}$, yellow blocks, size

$120.14 \times 0.08 \times 0.04 \mathrm{~mm}$, triclinic, P-1 (no. 2), $\mathrm{a}=4.5032$ (1) Á, $\mathrm{b}=11.4730$

13 (4) Á, c = 13.0278 (4) Á, $\alpha=80.483(1)^{\mathrm{o}}, \beta=84.692(1)^{\mathrm{o}}, \gamma=89.314(1)^{\mathrm{o}}$,

$14 \mathrm{~V}=666.97(3) \AA^{3}, \mathrm{Z}=2, \mathrm{D}_{\text {calcd }}=1.454 \mathrm{Mg} \mathrm{m}^{-3}, \mathrm{~F}(000)=300, \mu=2.28$

$15 \mathrm{~mm}^{-1}, \mathrm{~T}=123 \mathrm{~K} 10950$ measured reflections $\left(2 \theta_{\max }=144.4^{\circ}\right), 2601$

16 independent reflections $\left[\mathrm{R}_{\mathrm{int}}=0.025\right.$ ], 190 parameters, 3 restraints, $\mathrm{R}_{1}$ [for

$172515 \mathrm{I}>2 \sigma(\mathrm{I})]=0.027, \mathrm{wR}^{2}$ (for all data) $=0.070, \mathrm{~S}=1.07$, largest diff. 18 peak and hole $=0.25$ e $\AA^{-3} /-0.21 \mathrm{e} \AA^{-3}$.

19 CCDC 1961343 (5d) contains the supplementary crystallographic data for 20 this paper [27]. 
1 Acknowledgments Alaa A. Hassan is indebted to the AvH-Foundation for

2 the donation of a Shimadzu 408 IR instrument; The authors also thank

3 Egyptian Mission, Ministry of higher education, Egypt for its financial

4 support to Mrs. Lamiaa E. Abd El-Haleem during her accommodation at 5 the Karlsruhe Institute für Technology, Karlsruhe, Germany.

\section{References}

7 1. Aanandhi V, George S, Vaidhyalingam V (2008) Arkivoc xi: 187.

8 2. Suni MM, Nair VA, Joshua CP (2001) Tetrahedron 57:2003.

9 3. Hassan AA, Shawky AM, Shehatta HS (2012) J. Heterocycl. Chem. $10 \quad 49: 21$.

11 4. Katritzky AR, Khashab NM, Gromova AV (2006) Arkivoc iii: 226.

12 5. Hassan AA, Mohamed SK, Abdel-Latif FF, Mostafa SM, Mague JT, 13 Akkurt M, Abdel-Aziz M (2016) Tetrahedron Letters 57:345.

14 6. Hassan AA, El-Shaieb KM, Mohamed NK, Tawfeek HN, Bräse S, Nieger $15 \quad$ M (2014) Tetrahedron letters 55:2385.

16 7. Patel VM, Desai KR (2004) Arkivoc i: 123.

17 8. Matysiak J, Niewiadomy A (2006) Synthetic commun. 36:1621.

18 9. Aly AA, Brown AB, El-Emary TI, Ewas AMM, Ramadan M (2009) 19 Arkivoc i:150.

20 10. Eremkin AV, Ershov OV, Kayukov YS, Sheverdov VP, Nasakin OE, 21 Tafeenko VA, Nurieva EV (2006) Tetrahedron letters 47:1445. 
1 11. Hassan AA, El-Shaieb KM, Shaker RM, Döpp D (2005) Heteroatom

2 Chem. 16:12.

3 12. Hassan AA, Mourad AE, Abou-Zeid AH (2008) J. Heterocycl. Chem. $4 \quad 45: 323$.

5 13. Arafa WAA, Faty RAM, Mourad AK (2018) J. Heterocycl. Chem. $6 \quad 55: 1886$.

7 14. Hecht SM, Werner D, Traficante DD, Sundaralingam M, Prusiner P, Ito

$8 \quad$ T, Sakurai T (1975) J. Org. Chem. 40:1815.

9 15. Okuma K, Tsujimoto Y (2001) Heteroatom Chem. 12:259.

10 16. Hassan AA, Aly, AA, Mostafa SM (2018) Arkivoc iii: 200 .

11 17. Hassan, AA, Mohamed, NK, El-Shaieb, KMA, Tawfeek, HN, Bräse S,

12 Nieger M (2016) Arkivoc vi: 163.

13 18. Hassan AA, Aly, AA, Mohamed NK, El-shaieb, KM, Makhlouf, MM,

14 Bräse S, Nieger M, Brown AB (2018) Res. Chem. Intermed. 46:1571.

15 19. Hassan, AA, Ibrahim, YR, El-Sheref, EM, Bräse S (2016) J Heterocycl.

$16 \quad$ Chem. $53: 876$

17 20. Hassan, AA, Mourad, AE, Abou-Zied, AH (2007) J Heterocycl.

$18 \quad$ Chem. $44,1171$.

19 21. Kalinowski H-O, Berger S, Braun S (1984) ${ }^{13}$ C-NMR-Spektroskopie;

20 Georg Thieme Verlag: Stuttgart.

21 22. Gewald K, Schindler R (1990) J. prakt. Chem. 332:223. 
1 23. Grandi MDi, Olson M, Prashad AS, Bebernitz G, Luckay A, Mullen S,

2 Hu Y, Krishnamurthy G, Pitts K, O’Connell J (2010) J. Bioorg. Med.

$3 \quad$ Chem. Lett. 20:398.

4 24. Dolman SJ, Gosselin F, O'shea PD, Davies LW (2006) Org. Chem.

$5 \quad 71: 9548$.

6 25. Sheldrick GM (2015) Acta Crystallogra. A71:3.

7 26. Sheldrick GM (2015) Acta Crystallogra. C71:3.

8 27. These data can be obtained free of charge from The Cambridge

9 Crystallographic $\quad$ Data $\quad$ Centre via

$10 \quad$ www.ccdc.cam.ac.uk/data request/cif.

11 Figure Captions

12 Fig. 1 Molecular structure of 5d (displacement parameters are drawn at

$1350 \%$ probability level.

14 Scheme Captions

15 Scheme 1. Reactions of disubstituted thiosemicarbazids 1a-c with TCNE 2 16 in ethyl acetate.

17 Scheme 2. Reactions of $\mathbf{4 a - f}$ with TCNE 2 and formation of the products 18 5a-f.

19 Scheme 3. Mechanistic rationale for the formation of compounds 5a-f.

$20 \quad$ Figure 1 


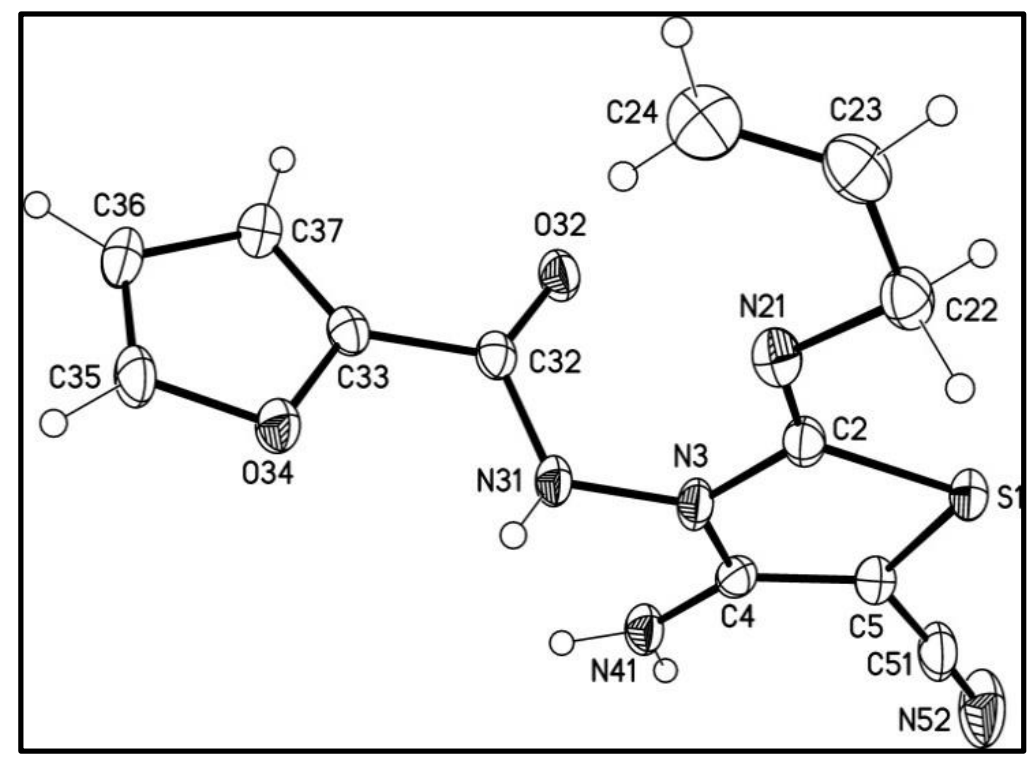

2 Scheme 1<smiles>[R]NC(=S)NNc1ccccc1</smiles>

$4 \quad$ Scheme 2<smiles>[R]CCC(=[R])NC(=O)Nn1c(N)c(C#N)sc1=N[R]</smiles>

6 
1 Table1, yields of synthetic products 5a-f in different solvents.

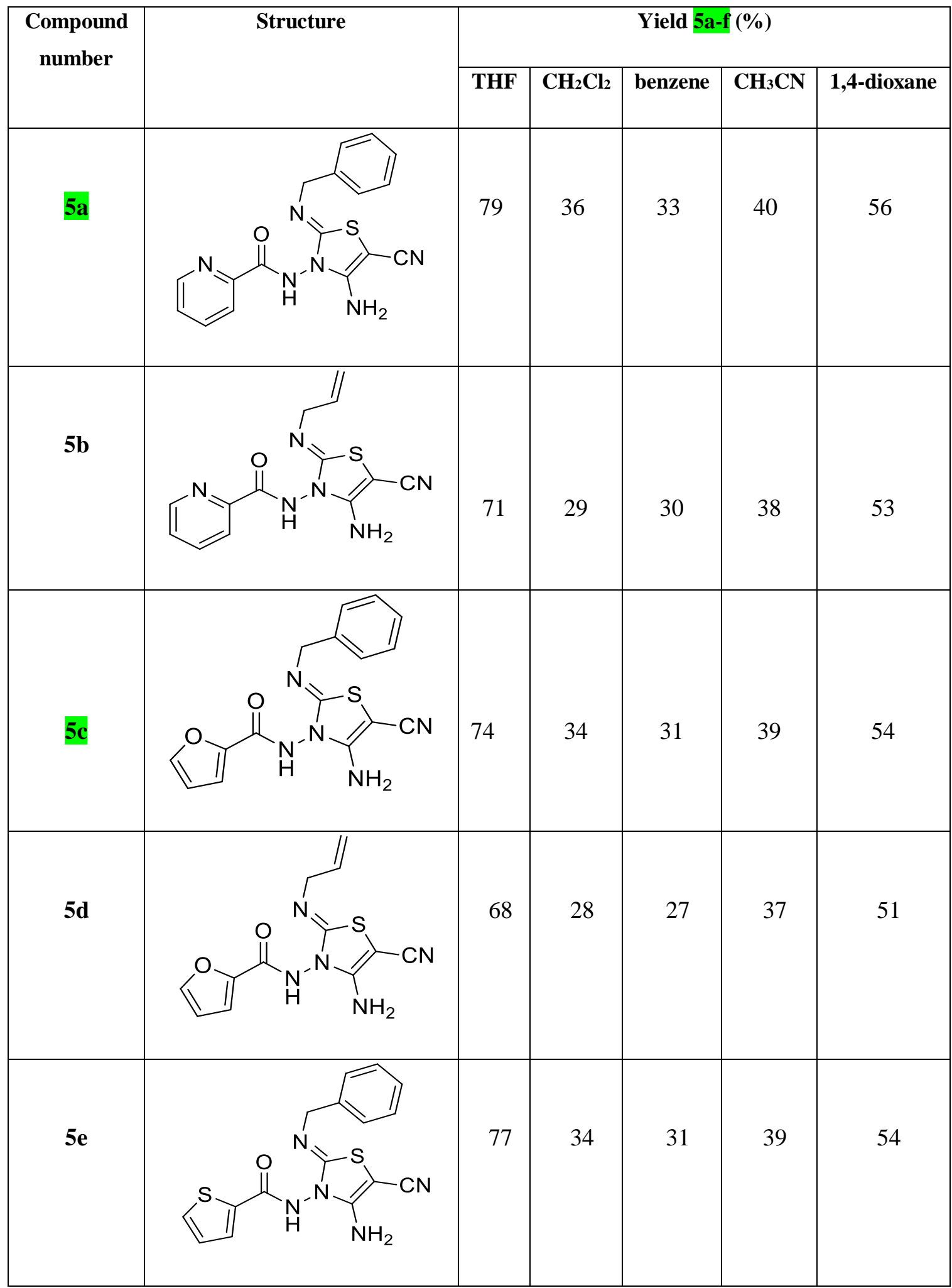




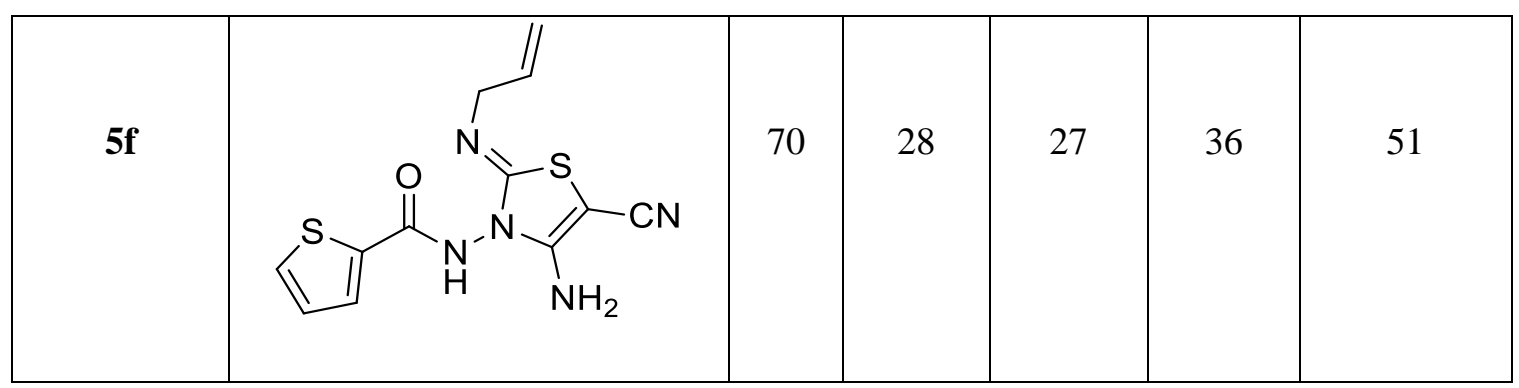

1

$2 \quad$ Scheme 3

$$
\text { 4a-f }+2 \rightleftharpoons[\text { CT-complex }] \rightleftarrows\left[\dot{4}^{+}+\ddot{2}^{-}\right] \longrightarrow[\dot{4}+2 \dot{\mathrm{H}}] \longrightarrow
$$
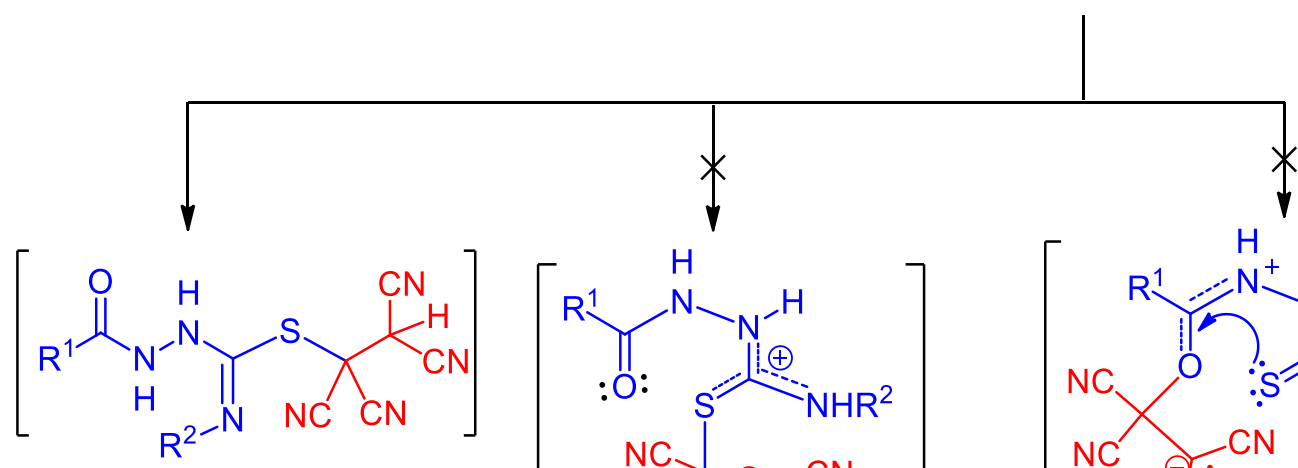

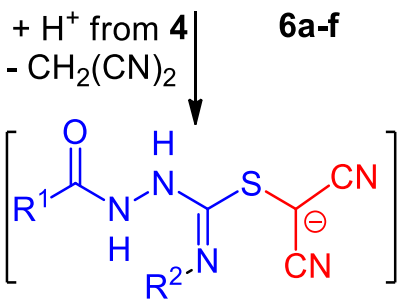
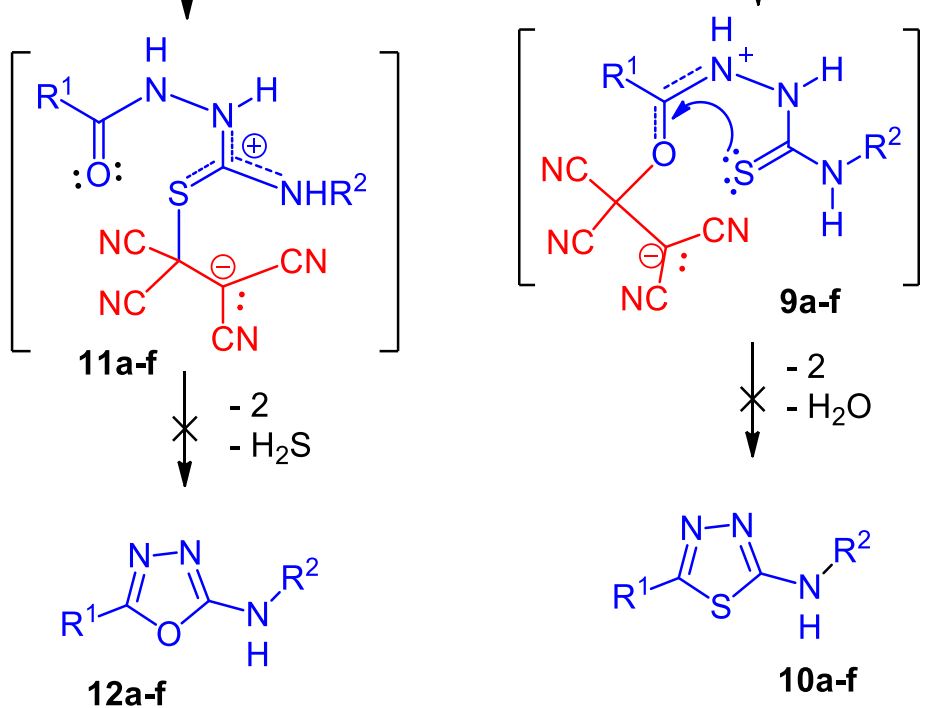

[a-f

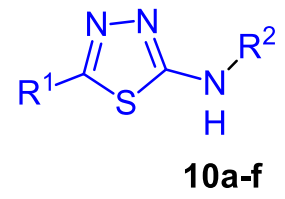<smiles>[R]N=c1sc(C#N)c(N)n1NC([R])=O</smiles> 


\section{Graphical abstract}

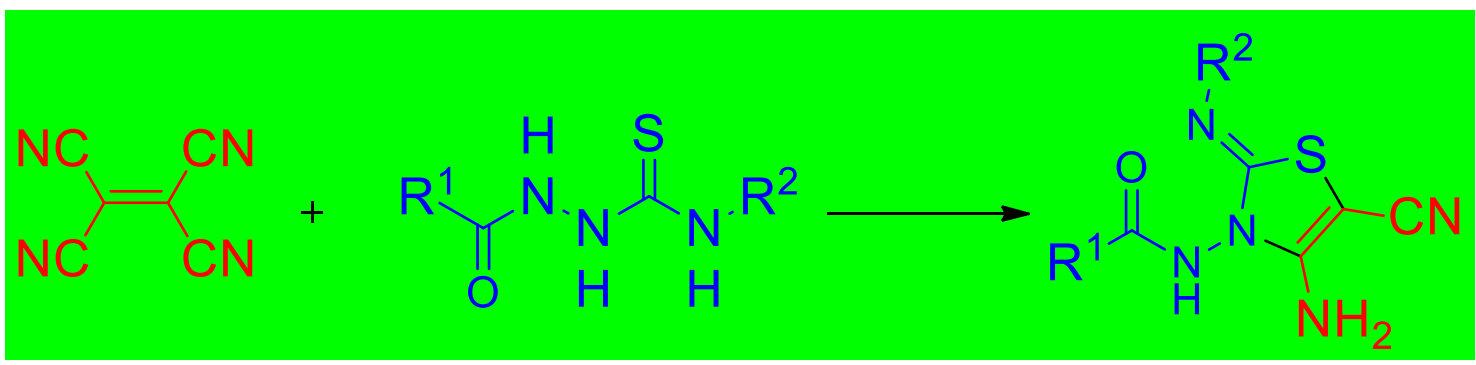

\section{Elemental analyses}

\begin{tabular}{|c|c|c|c|c|}
\hline No. & Compounds & $\begin{array}{c}\text { Chemical } \\
\text { Formula }\end{array}$ & Calculated & Found \\
\hline 1 & $\mathbf{5 a}$ & $\mathrm{C}_{17} \mathrm{H}_{14} \mathrm{~N}_{6} \mathrm{OS}$ & $\mathrm{C}, 58.27 ; \mathrm{H}, 4.03 ; \mathrm{N}$, & $\mathrm{C}, 58.33 ; \mathrm{H}, 4.10 ;$ \\
& & $23.98 ; \mathrm{S}, 9.15$ & $\mathrm{~N}, 24.04 ; \mathrm{S}, 9.22$ \\
\hline 2 & $\mathbf{5 b}$ & $\mathrm{C}_{13} \mathrm{H}_{12} \mathrm{~N}_{6} \mathrm{OS}$ & $\mathrm{C}, 51.99 ; \mathrm{H}, 4.03 ; \mathrm{N}$, & $\mathrm{C}, 52.05 ; \mathrm{H}, 4.11 ;$ \\
& & & $27.98 ; \mathrm{S}, 10.68$ & $\mathrm{~N}, 28.04 ; \mathrm{S}, 10.73$ \\
\hline 3 & $\mathbf{5 c}$ & $\mathrm{C}_{16} \mathrm{H}_{13} \mathrm{~N}_{5} \mathrm{O}_{2} \mathrm{~S}$ & $\mathrm{C}, 56.63 ; \mathrm{H}, 3.86 ; \mathrm{N}$, & $\mathrm{C}, 56.70 ; \mathrm{H}, 3.92 ;$ \\
& & & $20.64 ; \mathrm{S}, 9.45$ & $\mathrm{~N}, 20.69 ; \mathrm{S}, 9.51$ \\
\hline 4 & $\mathbf{5 d}$ & $\mathrm{C}_{12} \mathrm{H}_{11} \mathrm{~N}_{5} \mathrm{O}_{2} \mathrm{~S}$ & $\mathrm{C}, 49.82 ; \mathrm{H}, 3.83 ; \mathrm{N}$, & $\mathrm{C}, 49.88 ; \mathrm{H}, 3.89 ;$ \\
& & & $24.21 ; \mathrm{S}, 11.08$ & $\mathrm{~N}, 24.26 ; \mathrm{S}, 11.17$ \\
\hline 5 & $\mathbf{5 e}$ & $\mathrm{C}_{16} \mathrm{H}_{13} \mathrm{~N}_{5} \mathrm{OS}_{2}$ & $\mathrm{C}, 54.07 ; \mathrm{H}, 3.69 ; \mathrm{N}$, & $\mathrm{C}, 54.12 ; \mathrm{H}, 3.74 ;$ \\
& & & $19.70 ; \mathrm{S}, 18.04$ & $\mathrm{~N}, 19.74 ; \mathrm{S}, 18.10$ \\
\hline 6 & $\mathbf{5 f}$ & $\mathrm{C}_{12} \mathrm{H}_{11} \mathrm{~N}_{5} \mathrm{OS}_{2}$ & $\mathrm{C}, 47.20 ; \mathrm{H}, 3.63 ; \mathrm{N}$, & $\mathrm{C}, 47.25 ; \mathrm{H}, 3.69 ;$ \\
& & & $22.93 ; \mathrm{S}, 21.00$ & N, 22.98; S, 21.08 \\
\hline
\end{tabular}

